\title{
Effects of tilting the magnetic field in one-dimensional Majorana nanowires
}

\author{
Javier Osca, ${ }^{1, *}$ Daniel Ruiz, ${ }^{1}$ and Llorenç Serra ${ }^{1,2}$ \\ ${ }^{1}$ Institut de Física Interdisciplinària i de Sistemes Complexos IFISC (CSIC-UIB), E-07122 Palma de Mallorca, Spain \\ ${ }^{2}$ Departament de Física, Universitat de les Illes Balears, E-07122 Palma de Mallorca, Spain \\ (Received 14 March 2014; revised manuscript received 23 May 2014; published 5 June 2014)
}

\begin{abstract}
We investigate the effects that a tilting of the magnetic field from the parallel direction has on the states of a one-dimensional Majorana nanowire. Particularly, we focus on the conditions for the existence of Majorana zero modes, uncovering an analytical relation (the projection rule) between the field orientation relative to the wire, its magnitude, and the superconducting parameter of the material. The study is then extended to junctions of nanowires, treated as magnetically inhomogeneous straight nanowires composed of two homogeneous arms. It is shown that their spectrum can be explained in terms of the spectra of two independent arms. Finally, we investigate how the localization of the Majorana mode is transferred from the magnetic interface at the corner of the junction to the end of the nanowire when increasing the arm length.
\end{abstract}

DOI: 10.1103/PhysRevB.89.245405

PACS number(s): 73.63.Nm, 74.45.+c

\section{INTRODUCTION}

In 2003, Kitaev pointed out the usefulness of topological states for quantum computing operations [1]. Essentially, topological states are quantum states with a hidden internal symmetry [2]. They are usually localized close to the system edges or interfaces and their nonlocal nature gives them a certain degree of immunity against local sources of noise. A subset of this kind of states called Majorana edge states is attracting much interest in condensed matter physics [3-13]. Majorana states are effectively chargeless zero-energy states that behave as localized non-Abelian anyons. It is theorized that nontrivial phases arise from their mutual interchange, caused by their nonlocal properties [14,15]. Furthermore, these states have the property of being their own antistates, giving rise to statistical behavior that is neither fermionic nor bosonic. Instead, the creation of two Majorana quasiparticle excitations in the same state returns the system to its equilibrium state. This kind of quasiparticle inherits its name from Majorana who theorized the existence of fundamental particles with similar statistical properties [16].

Majorana states have been theoretically predicted in many different systems, and some of them have been realized experimentally. In particular, evidences of their formation at the ends of semiconductor quantum wires inside a magnetic field with strong spin-orbit interaction and in close proximity to a superconductor have been seen in Refs. [17-21]. Superconductivity breaks the charge symmetry creating quasiparticle states without a defined charge that are a mixture of electron and hole excitations. On the other hand, the spin-orbit Rashba effect is caused by an electric field perpendicular to the propagation direction that breaks the inversion symmetry of the system while the external magnetic field breaks the spin-rotation symmetry of the nanowire. The combined action of both effects makes the resulting state effectively spinless and, including superconductivity, also effectively chargeless and energyless [22-41].

This work addresses the physics of one-dimensional (1D) nanowires with varying relative orientations between the

\footnotetext{
*javier@ifisc.uib-csic.es
}

external magnetic field and the nanowire (see Fig. 1). This physics is of relevance, e.g., for the exchange of Majoranas on networks of $1 \mathrm{D}$ wires, where it has been suggested that Majoranas can be braided by manipulating the wire shapes and orientations [42-44]. The Hamiltonian of the system is expressed in the continuum and the analysis is performed using two complementary approaches: the complex band structure of the homogeneous wire and the numerical diagonalization for finite systems. The complex band structure allows a precise characterization of the parameter regions of the semi-infinite wire where Majoranas, if present, are not distorted by finite-size effects. On the contrary, numerical diagonalizations of finite systems, even though reflecting the same underlying physics, yield smoothened transitions between different physical regions of parameter space.

For the semi-infinite system, we uncover an analytical law limiting the existence of Majorana modes below critical values of the angles between the magnetic field and the nanowire. This law, referred to in this paper as the projection rule, is shown to be approximately valid in finite systems too. We find a correspondence of the finite-system spectrum with its infinite wire counterpart, explaining this way its distinctive features and regimes in simplest terms. Our work is related to Ref. [31], which proved the influence of a transverse component of the field to be consistent with the experimental observations of Ref. [17].

The results for the homogeneous nanowire are subsequently used to explain the spectrum of a junction of two nanowires with arbitrary angle. The junction is modeled as a nonhomogeneous straight nanowire with two regions characterized by different magnetic field orientations [see Fig. 1(b)]. While the magnetic field remains parallel to the nanowire in one arm, we study the spectrum variation when changing the magnetic field angles in the other. Similarities between the homogeneous and inhomogeneous nanowire spectra allow us to explain many of the features of the latter in terms of those of the former. Finally, we investigate the dependence with the distance of the magnetic interface (the corner of the junction) to the end of the nanowire, finding a transfer phenomenon where the Majoranas change localization from the interface for a short arm to the nanowire end as the arm length is increased. 
(a)

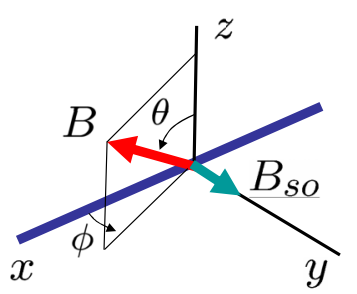

(b)

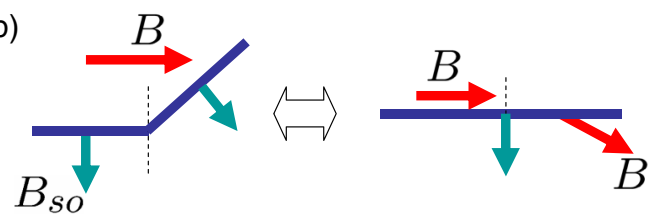

FIG. 1. (Color online) Sketches of the physical systems considered in this work. (a) Straight nanowire on the $x$ axis in a homogeneous magnetic field characterized by spherical angles $\theta$ and $\phi$. (b) Junction of two nanowires in a uniform magnetic field (left) represented as a straight nanowire with a magnetic inhomogeneity (right).

This work is organized as follows. In Sec. II, the physical model is introduced and Sec. III presents the above-mentioned projection rule. In Sec. IV, we discuss the spectrum of excited states of a homogeneous nanowire, while in Sec. V we address an inhomogeneous system representing a nanowire junction. We study changes in the spectrum due to the tilting (Sec. V A) and stretching (Sec. V B) of one of the junction arms. Finally, the conclusions of the work can be found in Sec. VI.

\section{PHYSICAL MODEL}

We assume a one-dimensional model of a semiconductor nanowire as a low-energy representation of a higherdimensional wire with lateral extension, when only the first transverse mode is active. The system is described by a Hamiltonian of the Bogoliubov-de Gennes kind

$$
\begin{aligned}
\mathcal{H}_{\mathrm{BdG}}= & \left(\frac{p_{x}^{2}}{2 m}+V(x)-\mu\right) \tau_{z} \\
& +\Delta_{B}\left(\sin \theta \cos \phi \sigma_{x}+\sin \theta \sin \phi \sigma_{y}+\cos \theta \sigma_{z}\right) \\
& +\Delta_{s} \tau_{x}+\frac{\alpha}{h} p_{x} \sigma_{y} \tau_{z},
\end{aligned}
$$

where the different terms are, in left to right order, kinetic, electric, and chemical potential, Zeeman, superconducting, and Rashba spin-orbit terms. The Pauli operators for spin are represented by $\sigma_{x, y, z}$ while those for isospin are given by $\tau_{x, y, z}$. Superconductivity is modeled as an s-wave superconductive term that couples different states of charge.

The superconductor term in Eq. (1) is an effective mean field approximation to a more complicated phonon-assisted attractive interaction between electrons. This interaction leads to the formation of Cooper pairs with breakup energy $\Delta_{s}$. Experimentally, superconductivity can be achieved by close proximity between the semiconductor nanowire and a metal superconductor. The semiconductor wire becomes superconducting when its width is smaller than the coherence length of the Cooper pairs. On the other hand, the Rashba spin-orbit term arises from the self-interaction between an electron (or hole) spin with its own motion. This self-interaction is due to the presence of a transverse electric field that is perceived as an effective magnetic field in the rest frame of the quasiparticle. This electric field can be induced externally but, usually, is a by-product of an internal asymmetry of the nanostructure. In the Hamiltonian (1), we have taken $\hat{x}$ as the orientation of the 1D nanowire while an effective spin-orbit magnetic field $\vec{B}_{\text {so }}$ pointing along $\hat{y}$ may be defined due to the coupling of the Rashba term with the $y$ component of the spin.

We consider the nanowire in an external magnetic field, giving spin splittings through the Zeeman term in Eq. (1). In this paper, we assume the magnetic field in arbitrary direction, including the possibility of being inhomogeneous in space for some setups. The direction of the magnetic field is parametrized by the spherical polar and azimuthal angles $\theta$ and $\phi$. These two angles are constant for a homogeneous wire [Fig. 1(a)] and they change smoothly from one to the other arm in a nanowire junction [Fig. 1(b)].

Summarizing, superconductor, Rashba spin-orbit, and Zeeman effects are parametrized in Eq. (1) by $\Delta_{s}, \alpha$, and $\Delta_{B}$, respectively. These parameters are taken constant because the nanowire is considered to be made of a homogeneous material. The only inhomogeneity allowed in certain cases is a change in the magnetic field direction at a single magnetic interface between two homogeneous regions.

Along this work the Hamiltonian of Eq. (1) is solved for homogeneous parameters in the infinite, semi-infinite, and finite wires, as well as for the inhomogeneous finite case, using different approaches. When a direct diagonalization of the Hamiltonian for a finite system is performed, soft potential edges and magnetic interface are used. The shape of the potential edges is modeled as Fermi-type functions centered on those edges. High potential is imposed outside the nanowire while low potential (usually zero) is assumed inside. When a magnetic interface is present, a smooth variation in the field angles is modeled in the same way. Specifically, those smooth functions read as

$$
\begin{gathered}
V(x)=V_{0}\left[1+\mathcal{F}\left(x ; x_{L}, s_{v}\right)-\mathcal{F}\left(x ; x_{R}, s_{v}\right)\right], \\
\theta(x)=\theta_{L}+\left(\theta_{R}-\theta_{L}\right)\left[1-\mathcal{F}\left(x ; x_{m}, s_{m}\right)\right], \\
\phi(x)=\phi_{L}+\left(\phi_{R}-\phi_{L}\right)\left[1-\mathcal{F}\left(x ; x_{m}, s_{m}\right)\right]
\end{gathered}
$$

for the potential and the field polar and azimuthal angles, respectively. The Fermi function $\mathcal{F}$ is defined as

$$
\mathcal{F}\left(x ; x_{0}, s\right)=\frac{1}{1+e^{\left(x-x_{0}\right) / s}} .
$$

In Eq. (2) $V_{0}$ is the value of the potential outside the nanowire, while $\theta_{L / R}$ and $\phi_{L / R}$ are the field angles at left and right of the magnetic interface. The potential left and right edges are centered on $x_{L}$ and $x_{R}$ and the magnetic interface is centered on $x_{m}$. Their softness is controlled by the parameters $s_{v}$ and $s_{m}$, where zero softness means a steep interface and a high value implies a smooth one.

The numerical results of this work are presented in special units obtained by taking $\hbar, m$, and the Rashba spin-orbit 
interaction $\alpha$ as reference values. That is, our length and energy units are

$$
\begin{aligned}
& L_{\mathrm{so}}=\frac{\hbar^{2}}{\alpha m}, \\
& E_{\mathrm{so}}=\frac{\alpha^{2} m}{\hbar^{2}} .
\end{aligned}
$$

\section{A PROJECTION RULE}

Let us consider a nanowire in a uniform magnetic field with $\theta=90^{\circ}$ and an arbitrary $\phi$ [see Fig. 1(a)]. A direct diagonalization of Eq. (1) for a finite length of the wire and $\phi=15^{\circ}$ yields the spectrum depicted in Fig. 2 as a function of the magnetic field intensity. A main feature of this figure is the existence of a Majorana mode, lying very near zero energy, but only for a particular range of values of the magnetic field. For the parameters of the figure, the Majorana mode is created around $\Delta_{B}=0.3 E_{\mathrm{so}}$ and destroyed in a rather abrupt way around $\Delta_{B}=E_{\mathrm{so}}$.

It is well known that Majorana wave functions decay to zero towards the nanowire interior. We can therefore analyze the creation and destruction of Majoranas in the semi-infinite system and use those results to understand the physics of Majoranas in a finite system. In this approach, we eliminate from the analysis the finite-size effects caused by the overlapping of the Majorana wave functions at both ends of a finite nanowire. Although it is obvious that for long enough wires the size effect becomes negligible, disentangling finite-size behavior from intrinsic Majorana physics using calculations of only finite systems is much less obvious.

Majorana mode creation has been understood as a phase transition of the lowest-excited state, signaled by the closing and reopening of a gap in the infinite nanowire band spectrum [23], as shown in Figs. 3(a) and 3(b). The phase transition follows in this case a well-known law, requiring high-enough fields for Majoranas to exist:

$$
\Delta_{B} \geqslant \sqrt{\Delta_{s}^{2}+\mu^{2}} .
$$

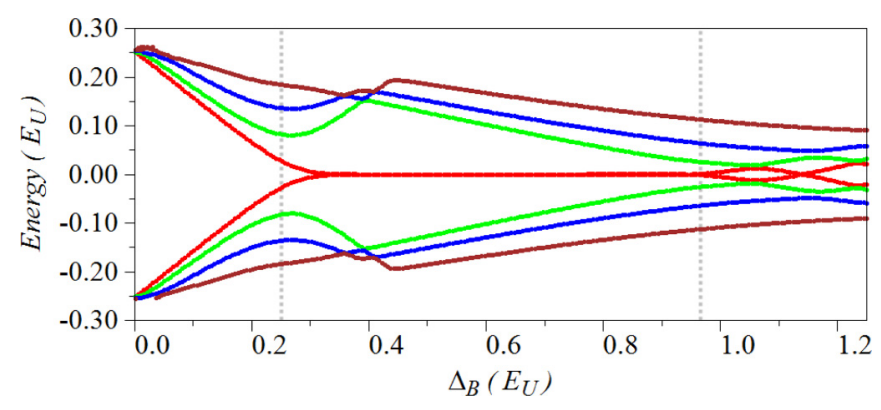

FIG. 2. (Color online) Spectrum of a finite-length nanowire with $L=50 L_{\mathrm{so}}$ as a function of the external magnetic field magnitude $\Delta_{B}$. Other nanowire parameters are $\Delta_{s}=0.25 E_{\mathrm{so}}$ and $\mu=0$. The magnetic field angles are $\theta=90^{\circ}$ and $\phi=15^{\circ}$. Only the eight states lying closer to zero energy are displayed. Note that a zero-energy Majorana mode is created at around $\Delta_{B}=0.3 E_{\mathrm{so}}$ and destroyed for values of $\Delta_{B}$ near one unit. The vertical lines (dots) indicate the onset and destruction of the Majorana mode as predicted by Eqs. (8) and (13), respectively.
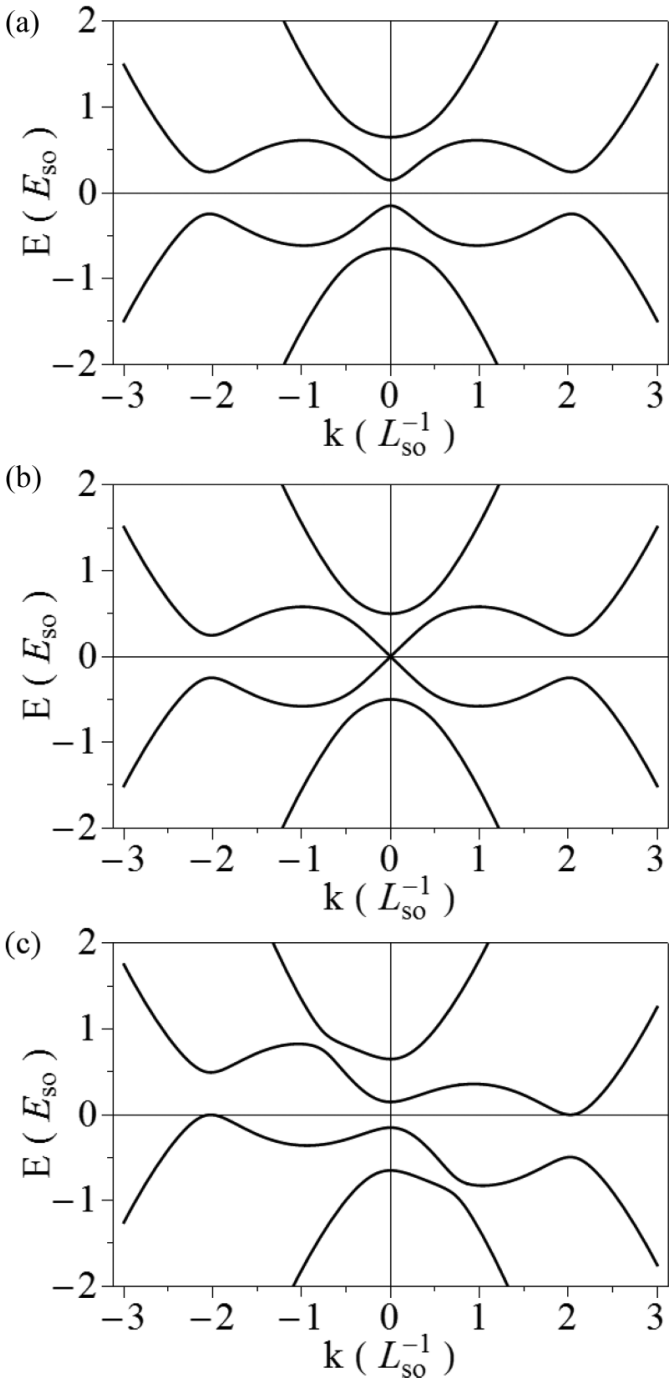

FIG. 3. Band structure of the infinite homogeneous nanowire with $\Delta_{s}=0.25 E_{\text {so }}$ and $\mu=0$ for (a) parallel field $(\theta, \phi)=\left(90^{\circ}, 0^{\circ}\right)$ with $\Delta_{B}=0.4 E_{\mathrm{so}}$, (b) the same as (a) but on the phase transition point $\Delta_{B}=0.25 E_{\mathrm{so}}$, (c) tilted field $(\theta, \phi)=\left(90^{\circ}, 38.68^{\circ}\right)$ with $\Delta_{B}=$ $0.4 E_{\text {so }}$.

Notice that, as mentioned, for the equality in Eq. (8) a gap closes for $k=0$ in Fig. 3(b). It is also worth stressing that Eq. (8) depends on the full Zeeman energy $\Delta_{B}$ and not just the partial contribution due to the parallel field $\Delta_{B} \sin \theta \cos \phi$, as one might naively assume.

In Ref. [38], Eq. (8) was derived, in an alternative way, from the analysis of the complex- $k$ solutions compatible with the boundary condition of a semi-infinite nanowire in a parallel field. This approach relies on the property that the complex band structure (allowing an imaginary part in $k$ ) of the homogeneous wire contains all the information about all possible eigenstates of any piecewise homogeneous wire. In general, an eigenstate of the infinite homogeneous wire with a given arbitrary $k$ can be expressed as

$$
\Psi^{(k)}\left(x, \eta_{\sigma}, \eta_{\tau}\right)=\sum_{s_{\sigma}, s_{\tau}} \Psi_{s_{\sigma} s_{\tau}}^{(k)} e^{i k x} \chi_{s_{\sigma}}\left(\eta_{\sigma}\right) \chi_{s_{\tau}}\left(\eta_{\tau}\right),
$$


where $\Psi_{s_{\sigma} s_{\tau}}^{(k)}$ are state amplitudes and the quantum numbers are $s_{\sigma}= \pm$ and $s_{\tau}= \pm$. In Eq. (9), $\eta=\uparrow, \downarrow$ indicate bivalued variables for spin $\left(\eta_{\sigma}\right)$ and isospin $\left(\eta_{\tau}\right)$. The corresponding spinors are denoted by $\chi(\eta)$ [38]. It is worth stressing here that analytical treatments in the limits of very weak and very strong Rashba couplings were discussed in Refs. [34,45]. We follow a general approach, at the price of being numerical.

The sharp semi-infinite wire with $x>0$ is obviously piecewise homogeneous, implying that the Majorana solution allowed by the existence of an edge at $x=0$ must be a linear superposition of the homogeneous nanowire eigenstates of complex wave number with $\operatorname{Im}(k)>0$, otherwise, it could not be a localized state. The resulting restriction is

$$
\sum_{k, \operatorname{Im}(k)>0} C_{k} \Psi_{s_{\sigma} s_{\tau}}^{(k)}=0,
$$

where the $C_{k}$ 's are complex numbers characterizing the superposition of state amplitudes. The allowed wave numbers are calculated solving the determinant

$$
\operatorname{det}\left\{H_{s_{\sigma} s_{\tau}, s_{\sigma}^{\prime} s_{\tau}^{\prime}}(k)-E \mathbb{1}\right\}=0,
$$

for $E=0$. In fact, the allowed $k$ 's can be calculated for any energy, but we are interested in particular in those at zero energy corresponding to Majorana solutions.

The wave-number dependence on magnetic field is depicted in Fig. 4(a) for a selected case. For a fixed energy $E$, there are always eight possible wave numbers, but only those with $\operatorname{Im}(k)>0$ are displayed in Fig. 4(a). In this representation, the closing of the $k=0$ gap in Fig. 3(b) corresponds to a node of
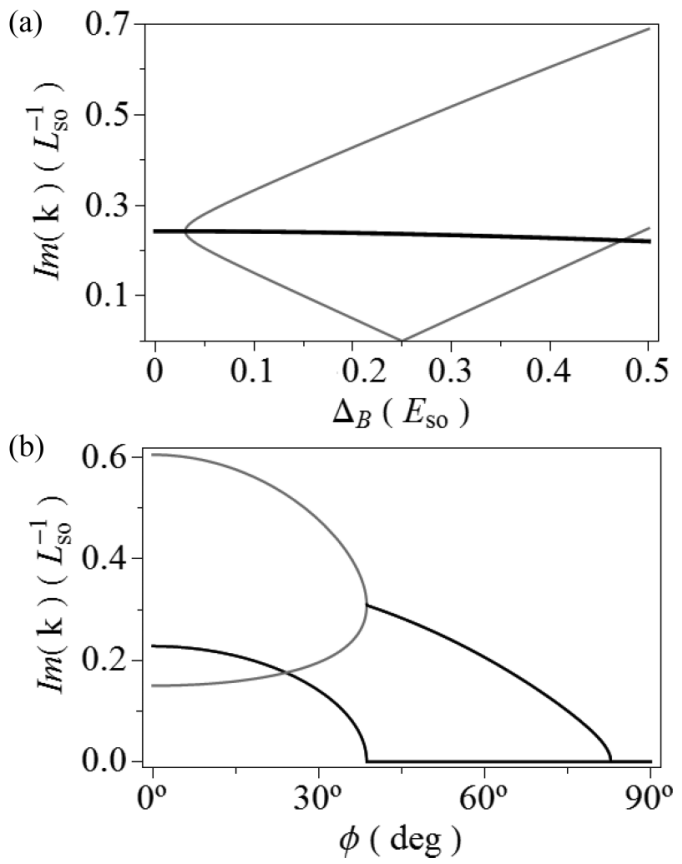

FIG. 4. Imaginary parts of the wave numbers (only positive ones) in an infinite homogeneous nanowire with $\Delta_{s}=0.25 E_{\text {so }}$ and $\mu=0$ as function of (a) the value of the longitudinally oriented magnetic field, (b) the azimuthal angle $\phi$ of a magnetic field with $\Delta_{B}=0.4 E_{\mathrm{so}}$ and polar angle $\theta=90^{\circ}$. Gray color is used for nondegenerate modes while black is indicating degeneracy with two or more modes actually having the same $\operatorname{Im}(k)$.
$\operatorname{Im}(k)$ in Fig. 4(a). In order to be able to hold a Majorana, a semi-infinite nanowire has to fulfill two simultaneous requirements. First, the nanowire must have four complex wave numbers with $\operatorname{Im}(k)>0$ allowed at zero energy; and second, a solution different from zero (nontrivial) must be possible for the $C_{k}$ 's in Eq. (10). That is, interpreting the state amplitudes $\Psi_{s_{\sigma} s_{\tau}}^{(k)}$ as a $4 \times 4$ matrix where the four $k$ 's correspond for instance to rows and the four spin-isospin values $\{++,+-,-+,--\}$ to columns, the condition for a nontrivial solution is

$$
\operatorname{det}\left\{\Psi_{s_{\sigma} s_{\tau}}^{(k)}\right\}=0 .
$$

In a parallel field, this condition is fulfilled only above a critical value of the magnetic field $\Delta_{B}^{(c)}=\sqrt{\Delta^{2}+\mu^{2}}$, but not under this quantity, thus leading to Eq. (8). Further details on the methodology can be found in Ref. [38]. Here, we want to use this approach to determine whether a similar condition on the field orientation, with critical values of the angles, exists or not.

Figure 4(b) shows the evolution of the wave numbers when increasing $\phi$ while maintaining $\theta=90^{\circ}$, i.e., maintaining the magnetic field in the plane formed by the nanowire direction and the effective spin-orbit magnetic field direction $\vec{B}_{\text {so }}$. This means that for $\phi=0^{\circ}$, the magnetic field is aligned with the nanowire, while for $\phi=90^{\circ}$ it is completely perpendicular to it and parallel to $\vec{B}_{\text {so }}$. In Fig. 4(b), care has been taken to choose a value of $\Delta_{B}$ that fulfills the Majorana condition for the parallel $\phi=0$ orientation Eq. (8). We can see that for $\phi=40.1^{\circ}$ two of the complex wave numbers become real, thus destroying the Majorana mode for azimuthal angles above this value.

The physical behavior implied by Fig. 4(b) is a sudden loss of the Majorana mode as the tilting angle $\phi$ exceeds a critical value, due to the system no longer having the required four evanescent modes with $\operatorname{Im}(k)>0$. The evanescent modes are lost because of the closing of the gap between states of opposite wave numbers $\left[k \approx \pm 2 L_{\text {so }}^{-1}\right.$ for the particular case shown in Fig. 3(c)]. We characterize next the dependence of the critical angle on $\Delta_{B}$ and $\Delta_{s}$. In Fig. 5(a), we can see a contour plot of $\operatorname{Im}(k)$ as a function of $\phi$ and the ratio $\Delta_{S} / \Delta_{B}$ for an external branch wave number [34], corresponding to the lower black line of Fig. 4(b). The values where $\operatorname{Im}(k)$ vanish separate the plot into two regions: the lower one where the Majorana is allowed and the upper (white) where no Majorana can exist. Although Eq. (11) can be solved analytically, the angles where $\operatorname{Im}\left[k\left(\phi, \Delta_{s} / \Delta_{B}\right)\right]$ vanishes can be obtained only numerically because $\phi$ appears as argument of sine and cosine functions and no isolation is possible. As a consequence, the values of $\phi$ where the wave number first reaches zero have been found numerically and are plotted in Fig. 5(c) against the test function $\arcsin \left(\Delta_{S} / \Delta_{B}\right)$. The perfect coincidence between the two results within computer precision demonstrates that a Majorana can not exist for angles such that $\sin \phi>\Delta_{s} / \Delta_{B}$, provided $\theta=90^{\circ}$.

Figure 5(b) shows a contour plot of $\operatorname{Im}(k)$ for an internal branch wave number [34], corresponding to the upper mode in Fig. 4(b). In this plot, the $\phi$ roots of $\operatorname{Im}(k)$ lie inside an upper and lower bounded region around $0.95 E_{\text {so }}<\Delta_{B}<$ $E_{\text {so }}$. In fact, two of the wave numbers become real in the white region of the contour plot. Note that this region lies 

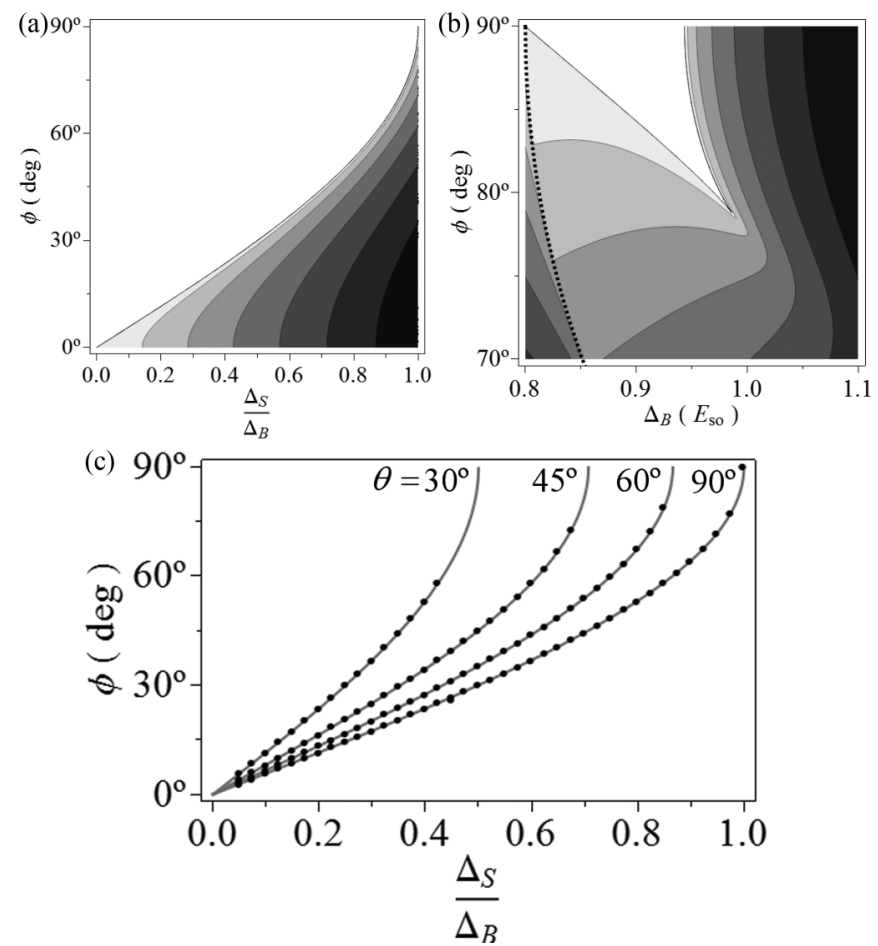

FIG. 5. (a) Contour plot of $\operatorname{Im}(k)$ for the external branch of the nanowire propagating bands. The horizontal axis contains the ratio $\Delta_{s} / \Delta_{B}$ and the vertical one the azimuthal angle $\phi$. The polar angle is fixed to $\theta=90^{\circ}$ and $\mu=0$. (b) Contour plot of $\operatorname{Im}(k)$ for the internal branch of the nanowire propagating bands. The horizontal axis shows $\Delta_{B}$ and the vertical one the azimuthal angle $\phi$. The polar angle is fixed to $\theta=90^{\circ}, \Delta_{s}=0.8 E_{\mathrm{so}}$, and $\mu=0$. (c) Plot of the azimuthal critical angle where $\operatorname{Im}(k)$ vanishes in the upper left panel as function of $\Delta_{s} / \Delta_{B}$ (points) checked against the projection-rule prediction (13). Aside from the $\theta=90^{\circ}$ case of the upper left panel, the figure also contains the comparison for other values of $\theta$. The value of the chemical potential can be taken arbitrarily since it is irrelevant for this comparison.

in the non-Majorana sector, above the transition discussed in Fig. 5(a) which is now signaled by the dotted line. Theoretically, the existence of this region determines two different fermionic regimes: one where a fermion mode at zero energy is constructed of plane waves with two complex and two real wave numbers and another one made of a full set of real wave numbers. Since we assume bound states in order to extrapolate the results to finite systems, these cases have no relevance to us. Nevertheless, the underlying causes for the existence of this region will be relevant in the study of the excited states of the finite nanowire. This will be further developed in Sec. IV.

Repeating the analysis for different polar angles $\theta$, as shown in Fig. 5(c), we conclude that the angular restriction for the existence of Majoranas is

$$
\Delta_{B} \sin \theta \sin \phi<\Delta_{s} .
$$

In other words, the projection of the magnetic field energy parameter into the spin-orbit effective magnetic field $\vec{B}_{\text {so }}$ needs to be smaller than the superconductor gap energy in order to have Majoranas in a semi-infinite wire. We refer to this condition as the projection rule. Notice that Eq. (13) is not a generalization of Eq. (8), but an additional law. Both Eqs. (8) and (13) have to be simultaneously met for the existence of a Majorana mode in a semi-infinite wire. Finding this projection rule using a complex band-structure approach is the main result of this work.

In general, the projection rule (13) yields an extra bound to be considered when identifying regions of Majoranas in parameter space. For instance, assuming fixed angles $(\theta, \phi)$ and varying $\Delta_{B}$, there is a lower bound on $\Delta_{B}$ from Eq. (8) and an upper bound from the projection rule. Analogously, if for a fixed $\Delta_{B}$ the Majorana is allowed by Eq. (8) at $\phi=0^{\circ}$ and and we increase $\phi$, the projection rule yields an upper bound on $\phi$. Therefore, as explained, both equations must be met simultaneously to obtain a Majorana mode. Furthermore, after some parameter testing we have determined that the projection rule is not affected by the value of the chemical potential $\mu$. This means that the overall dependence on $\mu$ for the existence of Majorana modes in the semi-infinite nanowire is completely covered by Eq. (8).

The disappearance of the Majorana when increasing $\phi$ is not a phase transition in the sense that no imaginary part of a mode wave number crosses zero in-between two regions with non-null values. As shown in Fig. 4(b) for the polar angle $\theta=90^{\circ}$, above the critical $\phi$ the value of $\operatorname{Im}(k)$ remains stuck at zero value. The main difference between the phase transition law in Eq. (8) and the projection rule (13) lies in the different type of gap closing for both cases. As shown in Fig. 3(b), the phase transition delimited by Eq. (8) is caused by a gap closing and reopening on a single wave number $k=0$ (labeled as interior branches of the spectrum). In the language of semiconductor band-structure physics, we may call this the closing of a direct gap. Oppositely, the projection rule is caused by the closing of an indirect gap for $k \approx \pm k_{f}$ (labeled as exterior branches of the spectrum), as shown in Fig. 3(c) for a selected case. This indirect gap remains closed if $\phi$ is further increased beyond a critical value [Fig. 4(b)], a sign of the metallic character of the new phase.

We have checked these laws against the direct numerical diagonalization for a finite nanowire, finding a reasonable agreement as shown in Figs. 2 and 6. In Fig. 2, the magnetic field orientation is kept fixed to a tilted orientation while the field magnitude is changed and in Fig. 6 the magnitude is fixed while the orientation is changed. The main difference between the precise laws for the semi-infinite model and the finite-system results is in the smoothness of the spectrum evolution around the transition points. While in the semiinfinite model the transition between fermionic modes to Majorana modes and vice versa happens at a single point in the parameter space, in the finite system we can see these transitions smoothed. This occurs due to the finite-size effects, i.e., the little overlap of Majoranas on opposite ends of the nanowire. Furthermore, while Majoranas lie at exactly zero energy in the semi-infinite model, this small interaction makes the finite system Majoranas to have a finite small energy $\epsilon$.

A close inspection of the projection rule (13) reveals that there exist critical values for $\theta$ and $\phi$ such that if they are not surpassed, a Majorana is always allowed, independently of the value of the other angle [provided Eq. (8) is fulfilled], that is, below the critical angle a projection into $\vec{B}_{\text {so }}$ is never high 

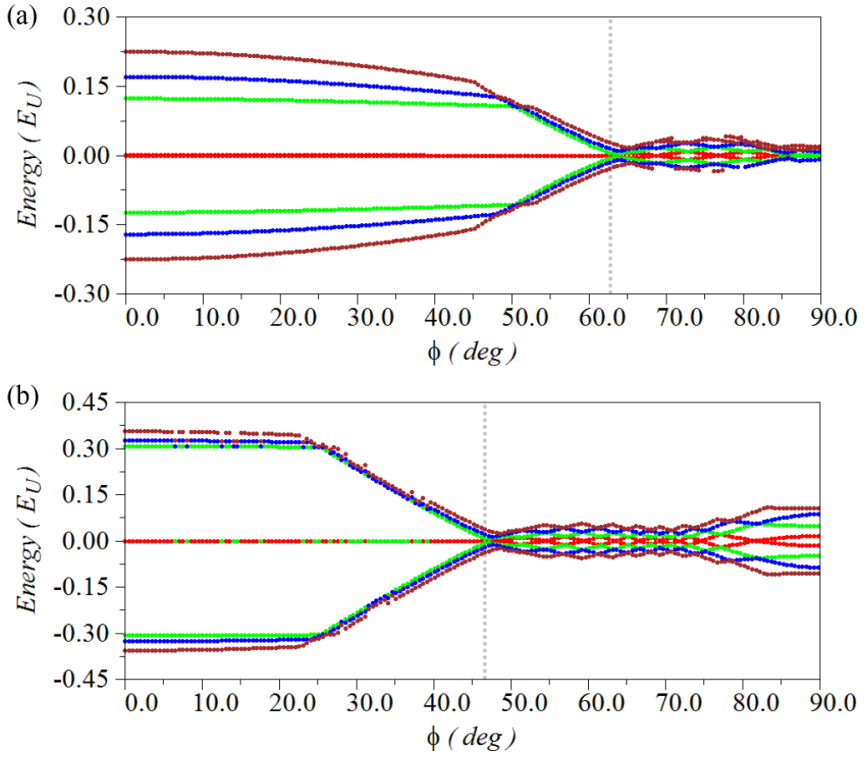

FIG. 6. (Color online) (a) Spectrum of a nanowire of length $L=$ $50 L_{\mathrm{so}}$ with $\Delta_{s}=0.8 E_{\mathrm{so}}$ and $\mu=0$ as a function of $\phi$ with a fixed $\theta=90^{\circ}$ and $\Delta_{B}=0.9 E_{\mathrm{so}}$. Note the spectrum change at the angle predicted by the projection rule (dotted line) as well as the spectrum collapse for values of $\phi$ close to $90^{\circ}$. For values of $\phi$ above $90^{\circ}$, the spectrum is given by the mirror image of the shown values. (b) The same as (a) but for a fixed magnetic field value $\Delta_{B}=1.1 E_{\mathrm{so}}$. Note that for values of $\phi$ close to $90^{\circ}$, now the two modes closer to zero are fermionic modes separated from each other by an energy gap.

enough to break the Majorana. In practice, if the Majorana is allowed by Eq. (8), it will survive for any $\phi$ provided $\theta<\theta_{c}$ or, alternatively, for any $\theta$ provided $\phi<\phi_{c}$. These critical angles are

$$
\theta_{c}=\phi_{c}=\arcsin \left(\frac{\Delta_{s}}{\Delta_{B}}\right)
$$

Tilting the magnetic field has been investigated in the experiment of Ref. [17], where the variation of differential conductance when rotating the magnetic field in the $x z$ and $y z$ planes (with the definitions of Fig. 1) has been followed in detail. A transition angle from presence to absence of zero bias anomaly is observed for $y z$ rotation, while no transition is found for $x z$ rotation. This is fully consistent with the projection rule [31], but the observed transition angle for $y z$ rotation seems to be smaller by a factor $\approx 2$ with respect to the theoretical prediction (14). We think this may be due to differences between our closed nanowire and the necessarily open experimental one, as well as to temperature effects [31]. In any case, an experimental confirmation of the critical angles in the sense of Eq. (14) would lend additional support to the Majorana scenario.

\section{EXCITED STATES}

While in the preceding section we focused on the physics of the Majoranas at zero energy, comparing semi-infinite and finite nanowires, in this section we address the spectrum of excited states. The main effect of the boundary conditions is to allow only a discrete set of wave numbers instead of
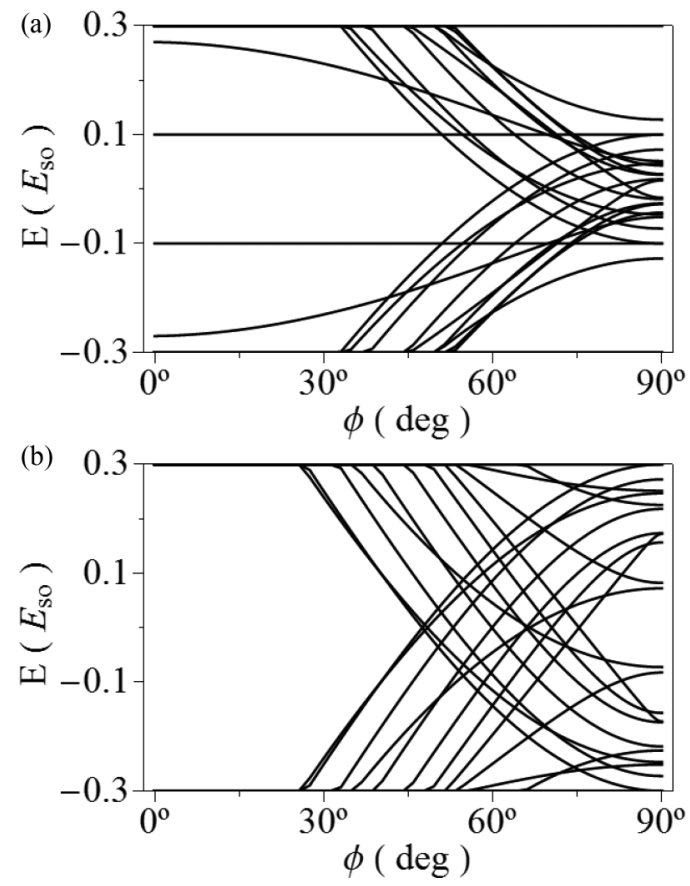

FIG. 7. Spectrum obtained from the homogeneous nanowire band energies at selected wave numbers, displayed as tracers as a function of $\phi$. Panel (a) corresponds to the same parameters of Fig. 6(a). Note that qualitatively similar regions occur for increasing angles in both figures. In particular, the spectrum nearly collapses for values of $\phi$ close to $90^{\circ}$. Panel (b) shows the same as (a) but for the parameters of Fig. 6(b). Note that for values of $\phi$ close to $90^{\circ}$, the two lower states (closer to zero energy) are fermionic modes separated by a gap as in Fig. 6(b).

a continuous one. What we have done is sketch the finite nanowire spectrum by selecting wave numbers at regular intervals and tracking the evolution of their energy levels with an increasing angle $\phi$. For these examples, we maintain the polar angle $\theta=90^{\circ}$ because this is the most physically interesting configuration due to the possibility of aligning external and spin-orbit magnetic fields; nevertheless, analogous plots can be done for different values of $\theta$. The resulting spectrum, shown in Fig. 7, explains the main features of the numerical diagonalization results of Fig. 6 for the same parameters.

In principle, we could also set the boundary conditions exactly as we did in Eq. (10), but we have found this approach impossible to follow on a practical level. The resulting set of equations reads as

$$
\begin{gathered}
\sum_{k} C_{k}^{(L)} \Psi_{s_{\sigma} s_{\tau}}^{(k)} e^{-i k L / 2}=0, \\
\sum_{k} C_{k}^{(R)} \Psi_{s_{\sigma} s_{\tau}}^{(k)} e^{i k L / 2}=0,
\end{gathered}
$$

where $C_{k}^{(L)}$ and $C_{k}^{(R)}$ are the coefficients at the left and right nanowire ends, respectively. Basically, the resulting matrix from Eq. (15) is ill defined since it contains very large and very small matrix elements.

The spectra of both panels of Fig. 7 can be divided into three different regions depending on the angle $\phi$. First, for low values of $\phi$ there is a region where a Majorana mode exists 

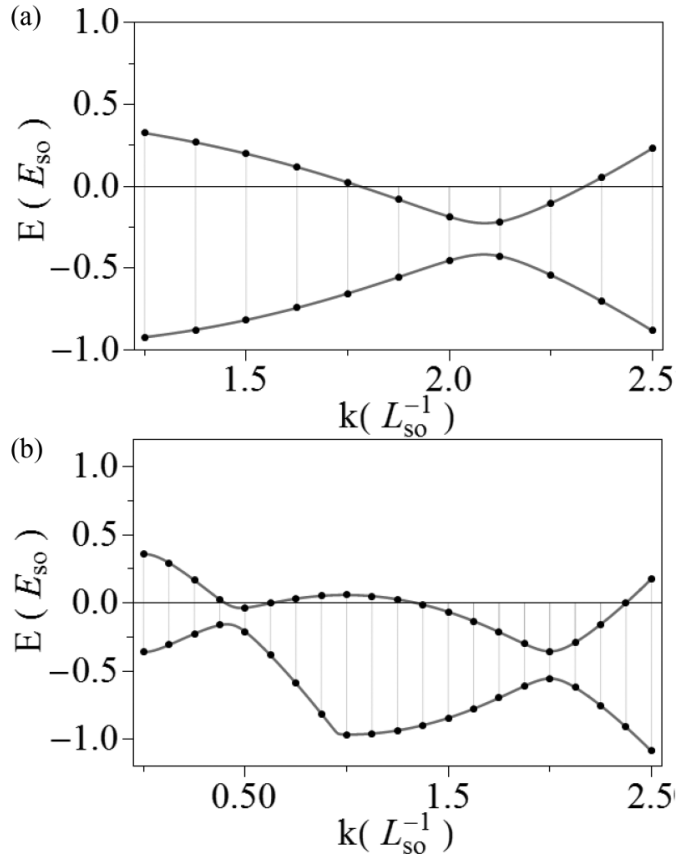

FIG. 8. Approximate spectrum of a finite nanowire in a particular configuration of the external magnetic field. (a) With only two positive real wave numbers. (b) With four positive real wave numbers.

and is topologically protected. In Fig. 7, the Majorana is not seen since only excited states of real wave number are shown, but we can see the corresponding gap. For values of $\phi$ above those determined by the projection rule, the Majorana mode is destroyed and we can see a region of many level crossings. This behavior of the spectrum is explained by the gap closing of the external branches of the conduction band noticing that in the finite model only some discrete values are allowed, as sketched in Fig. 8. Finally, for higher angular values, the region of zero crossings finishes and a third region arises with two possible behaviors.

As shown in Figs. 6 and 7 for high $\phi$ angles (near $90^{\circ}$ ), depending on the parameters the spectrum either opens a gap or collapses near zero energy. The behavior depends on the way the internal branches of the band cross the zero-energy value for those angles. The internal branches of the band can cross the zero-energy level for high angles in one $k>0$ point, like in Fig. 8(a), thus leading (jointly with the external branch crossing point) to four real and four complex wave numbers or, alternatively, the interior branch can cross zero energy in more than one $k>0$ point, like in Fig. 8(b), leading to wave functions characterized by eight real wave numbers. In the latter case, there is a wave-number range where the band spectrum lies very close to zero energy, yielding this way a collapse of the finite wire spectrum. The particular set of parameters where one or the other situation happens depends on the behavior of the internal branches of the band structure and it is not as easily predictable as the behavior of the external branches that led to the projection rule. The region of values where this collapse arises coincides with the region where the allowed solutions at zero energy are made of real wave numbers only and it was already presented in Fig. 5(a) for the $\mu=0$ case.

\section{MAGNETIC INHOMOGENEITY MODELS}

In this section, we explore the physics of a junction of two straight nanowires with a certain angle in presence of a homogeneous magnetic field parallel to one of the arms, as sketched in Fig. 1(b). We assume a representation of the system as a single straight $1 \mathrm{D}$ nanowire containing a magnetic interface. The inhomogeneity separates two homogeneous regions with different directions (but the same magnitude) of the external magnetic field. The system is solved by numerical diagonalization, assuming a soft magnetic interface, interpreting the results by comparing with the homogeneous nanowire discussed in the preceding section. We focus on two specific effects: tilting and stretching of one of the two junction arms. Other nanowire junction properties have been discussed in Refs. [39,46,47].

\section{A. Arm tilting}

The magnetic field is aligned with the left arm and the spectrum of the nanowire is computed for varying orientations of the field in the the right arm (see Fig. 9). As mentioned, this model represents under certain approximations a bent nanowire in a homogeneous magnetic field. It was shown in Ref. [48] that bent nanowires can be approximated by 1D models with a potential well simulating the effect of the bending. Here, we have only considered the magnetic field change of direction as the main inhomogeneity source, disregarding the electrical potential effects of the bending.

The spectrum of the inhomogeneous nanowire can be explained in terms of the homogeneous one for a tilted magnetic field. Figure 9 compares the inhomogeneous (upper)
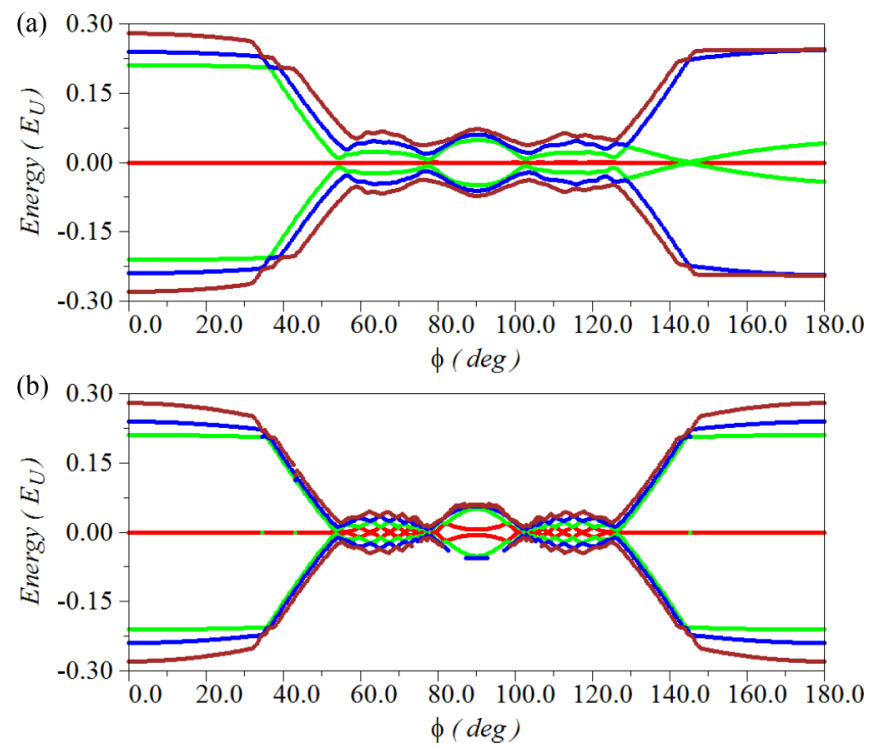

FIG. 9. (Color online) (a) Nanowire spectrum for $\Delta_{s}=0.8 E_{\text {so }}$ and $\mu=0$ with a magnetic inhomogeneity at its center as a function of the tilting angle $\phi$. On the left side of the nanowire the magnetic field is parallel, while on the right side its angles are $\left(\theta=90^{\circ}, \phi\right)$. The magnetic field strength is constant in both sides and equal to $\Delta_{B}=E_{\mathrm{so}}$. (b) Spectrum of a nanowire in a homogeneous magnetic field with angles $\left(\theta=90^{\circ}, \phi\right)$ and with the rest of parameters as in (a). 


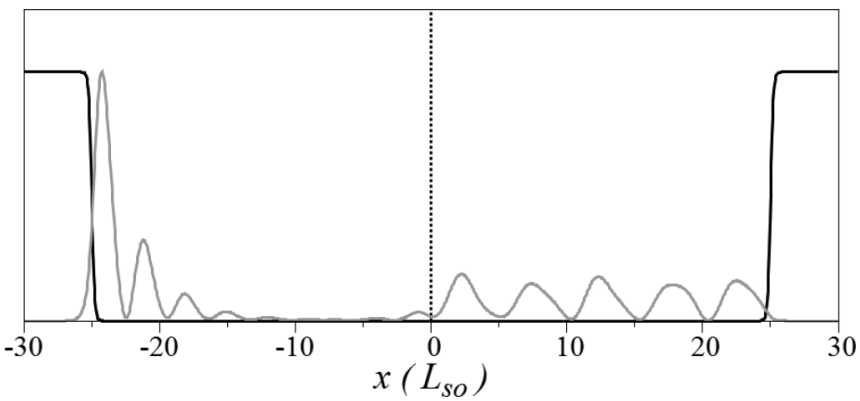

FIG. 10. Majorana density function of an inhomogeneous nanowire similar to the one described in Fig. 9(a). The magnetic field azimuthal angles in the two arms are $\phi_{L}=0^{\circ}$ and $\phi_{R}=90^{\circ}$, while all along the nanowire it is $\theta=90^{\circ}$. Other parameters are $\Delta_{B}=0.4$, $\Delta_{s}=0.25$.

with the homogeneous (lower) nanowire spectrum for the same set of parameters, showing that both results share the same essential features. More precisely, three $\phi$ regions can be found in both cases, but with two main differences. First, while for the homogeneous nanowire increasing $\phi$ leads to the destruction of the Majoranas on both ends, for the inhomogeneous nanowire only the localization of the right side Majorana is destroyed, leading to a spread state (Fig. 10). This spread state retains characteristic Majorana properties such as a nearly zero eigenenergy [Fig. 9(a)] and the typical relation of wave-function components. As a consequence, the bent junction holds only one localized Majorana mode in the left side of the inhomogeneity. The density of the Majorana for the inhomogeneous nanowire is shown in Figs. 10 and 11 for selected values of the parameters.

A second difference between upper and lower panels of Fig. 9 is that the spectrum for the inhomogeneous nanowire is not symmetric with respect to $\phi=90^{\circ}$, in contrast with the homogeneous nanowire. A zero-energy crossing localized in the inhomogeneity interface arises at $\phi=145^{\circ}$ for the selected parameters in Figs. 9(a) and 11. The corresponding bound state originates in the second excited state of the system and it is not Majorana in nature. Furthermore, this localized state is caused completely by the magnetic inhomogeneity and has no relationship with the localized states found in the bending region in Ref. [48] because we have disregarded those effects.

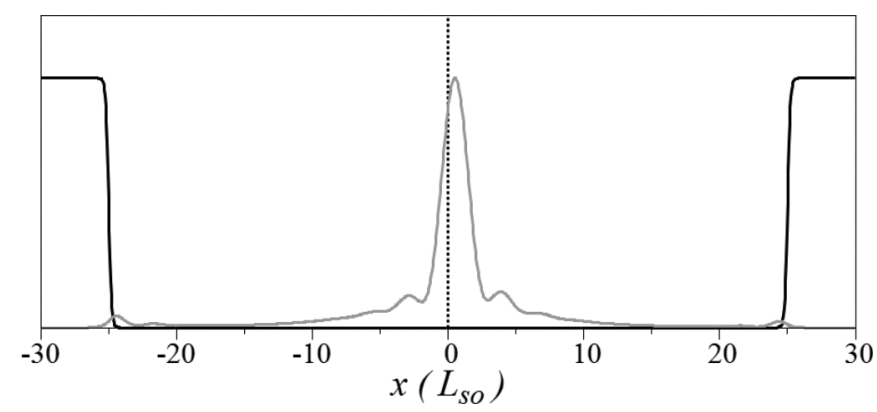

FIG. 11. Density function of the first excited state of the inhomogeneous nanowire of Fig. 9(a). This state becomes localized at the magnetic inhomogeneity for an azimuthal angle $\phi=145^{\circ}$ (and polar angle $\theta=90^{\circ}$ ).
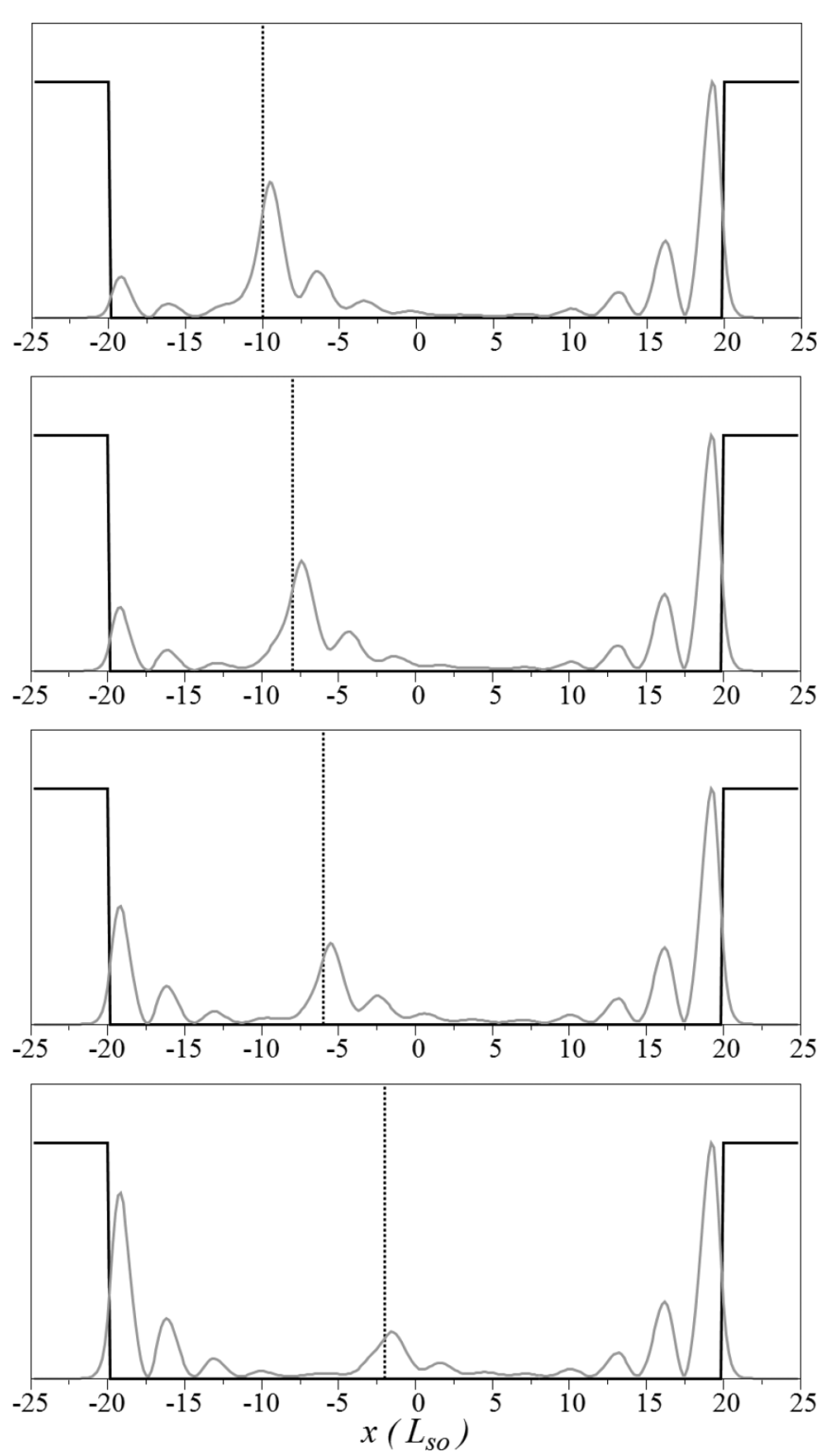

FIG. 12. Density distributions of the Majorana mode in a finite nanowire with a magnetic inhomogeneity. We used $\Delta_{s}=0.25 E_{\mathrm{so}}$, $\mu=0$, and a magnetic field of magnitude $\Delta_{B}=0.4 E_{\mathrm{so}}$ oriented parallel in the left side of the magnetic interface and antiparallel in the right side. In each panel, the potential well and the position of the magnetic interface are shown. The latter corresponds to a Fermi-type function whose position shifts to the right following the sequence from upper to lower panels.

Although we know these states are related with the magnetic inhomogeneity, a deep understanding of their causes and the particular set of parameters leading to their enhancement or quenching is yet to be understood.

\section{B. Arm stretching}

We study now the behavior of the Majorana modes in the nanowire as a function of the inhomogeneity distance to the nanowire end. The magnetic field directions are fixed at $\theta=90^{\circ}, \phi=0^{\circ}$ on the left end and $\theta=90^{\circ}, \phi=180^{\circ}$ on the right end of the nanowire. This is a particularly interesting 


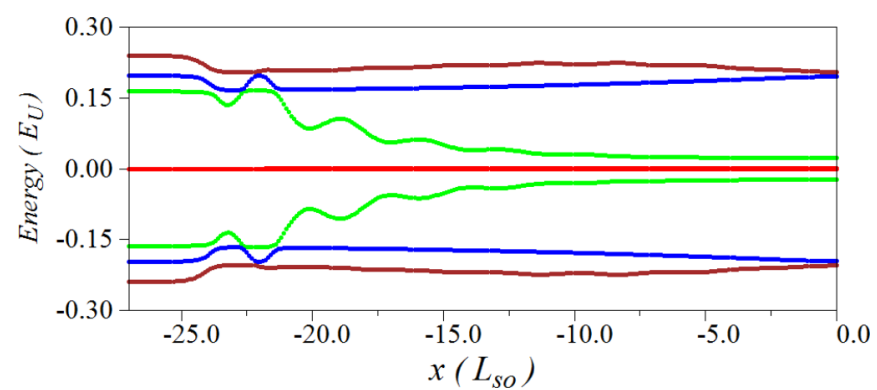

FIG. 13. (Color online) Spectrum of the nanowire described in the caption of Fig. 12 as a function the magnetic interface position.

configuration as it is the only setup where both ends lie inside a longitudinal magnetic field, apart from the homogeneous case. This way, all the observed effects must be caused by the inhomogeneity and its distance with respect to the left nanowire end.

Figure 12 shows the probability densities of the zero-energy state at different positions of the magnetic interface with respect to the left side of the nanowire. From upper to lower panels of Fig. 12 we may follow the evolution as the distance of the magnetic interface to the left end of the system is increased. Most remarkably, for short distance, the left Majorana is not peaked on the left end, but remains stuck on the magnetic interface (upper panels). If the distance is increased, however, the Majorana is eventually transferred to the left nanowire end after some critical distance (lower panels). This transfer is seen as a smooth decrease of the density maximum at the magnetic interface accompanied by an increase at the left end. Finally, when the interface is on the middle point of the nanowire, both Majoranas are located at their corresponding ends. It is also worth noticing that this Majorana transfer does not imply a departure of the mode from zero energy because the Majorana on the other end of the nanowire is not affected (see Fig. 13). Additionally, the transfer phenomenon is not caused by finite-size effects since we have checked that it happens for the same characteristic distance when the right end is further displaced to the right.

\section{CONCLUSIONS}

In this work, we have studied the spectra of 1D nanowires for arbitrary orientation of the magnetic field, focusing in particular on the conditions leading to a Majorana mode. This study has been realized from different perspectives and methods in an effort to explain the variety of observed phenomena. We have combined the complex band-structure techniques of infinite homogeneous nanowires with numerical diagonalizations of finite systems.

We have demonstrated an additional condition, aside from the well-known topological transition law, that needs to be taken into account in order to predict the regimes of existence of Majorana modes with tilted fields. We have named this additional condition the projection rule. The projection rule predicts an upper bound on the magnetic field at which Majoranas are to be found in a 1D wire with tilted field. We have stressed that the topological transition law with tilted fields depends on the full-field Zeeman energy and not just on the contribution of the magnetic field component parallel to the wire. When the topological law is fulfilled, the projection rule leads to critical values of the field angles $\theta_{c}$ and $\phi_{c}$, such that a Majorana mode is always found for any $\phi$ provided $\theta<\theta_{c}$ or, alternatively, for any $\theta$ provided $\phi<\phi_{c}$.

We have extended our analysis to nanowire junctions with an arbitrary angle, modeled as magnetically inhomogeneous nanowires, explaining most of their properties in terms of the behavior of its homogeneous parts. We have focused, particularly, on the role of tilting and stretching of one of the junction arms. We also reported the existence of a bound non-Majorana state located on the magnetic inhomogeneity. Finally, we have studied the Majorana transfer phenomenon as the distance of the magnetic inhomogeneity to the nanowire end is increased. Testing these predictions would require experiments of nanowires in inhomogeneous magnetic fields. Alternatively, it has been suggested in this work that a bent nanowire in a homogeneous field should display similar phenomena, while being more feasible in practice. As an interesting continuation of this work we are presently analyzing the validity of the projection rule in higher-dimensional nanowires, where the transverse degrees of freedom require a multimode description of the electronic states.

\section{ACKNOWLEDGMENT}

This work was funded by MINECO-Spain (Grant No. FIS2011-23526), CAIB-Spain (Conselleria d'Educació, Cultura i Universitats), and FEDER.
[1] A. Y. Kitaev, Ann. Phys. (NY) 303, 2 (2003).

[2] I. Affleck, T. Kennedy, E. H. Lieb, and H. Tasaki, Phys. Rev. Lett. 59, 799 (1987).

[3] F. Wilceck, Nat. Phys. 5, 614 (2009).

[4] X. L. Qi and S. C. Zhang, Rev. Mod. Phys. 83, 1057 (2011).

[5] J. Alicea, Rep. Prog. Phys. 75, 076501 (2012).

[6] M. Leijnse and K. Flensberg, Semicond. Sci. Technol. 27, 124003 (2012).

[7] C. W. J. Beenakker, Annu. Rev. Condens. Matter Phys. 4, 113 (2013).

[8] T. D. Stanescu and S. Tewari, J. Phys.: Condens. Matter 25, 233201 (2013).
[9] M. Franz, Nat. Nanotechnol. 8, 149 (2013).

[10] L. Fu and C. L. Kane, Phys. Rev. Lett. 100, 096407 (2008).

[11] A. R. Akhmerov, J. Nilsson, and C. W. J. Beenakker, Phys. Rev. Lett. 102, 216404 (2009).

[12] Y. Tanaka, T. Yokoyama, and N. Nagaosa, Phys. Rev. Lett. 103, 107002 (2009).

[13] K. T. Law, P. A. Lee, and T. K. Ng, Phys. Rev. Lett. 103, 237001 (2009).

[14] C. Nayak, S. H. Simon, A. Stern, M. Freedman, and S. Das Sarma, Rev. Mod. Phys. 80, 1083 (2008).

[15] J. K. Pachos, Introduction to Topological Quantum Computation (Cambridge University Press, Cambridge, UK, 2012). 
[16] E. Majorana, Nuovo Cimento 14, 171 (1937).

[17] V. Mourik, K. Zuo, S. Frolov, S. Plissard, E. Bakkers, and L. Kouwenhoven, Science 336, 1003 (2012).

[18] M. T. Deng, C. L. Yu, G. Y. Huan, M. Larsson, and P. Caroff, Nano Lett. 12, 6414 (2012).

[19] L. P. Rokhinson, X. Liu, and J. K. Furdyna, Nat. Phys. 8, 795 (2012).

[20] A. Das, Y. Ronen, Y. Most, Y. Oreg, M. Heiblum, and H. Shtrikman, Nat. Phys. 8, 887 (2012).

[21] A. D. K. Finck, D. J. Van Harlingen, P. K. Mohseni, K. Jung, and X. Li, Phys. Rev. Lett. 110, 126406 (2013).

[22] R. M. Lutchyn, J. D. Sau, and S. Das Sarma, Phys. Rev. Lett. 105, 077001 (2010)

[23] Y. Oreg, G. Refael, and F. von Oppen, Phys. Rev. Lett. 105, 177002 (2010).

[24] T. D. Stanescu, R. M. Lutchyn, and S. Das Sarma, Phys. Rev. B 84, 144522 (2011).

[25] K. Flensberg, Phys. Rev. B 82, 180516 (2010).

[26] A. C. Potter and P. A. Lee, Phys. Rev. Lett. 105, 227003 (2010).

[27] A. C. Potter and P. A. Lee, Phys. Rev. B 83, 094525 (2011).

[28] S. Gangadharaiah, B. Braunecker, P. Simon, and D. Loss, Phys. Rev. Lett. 107, 036801 (2011).

[29] R. Egger, A. Zazunov, and A. L. Yeyati, Phys. Rev. Lett. 105, 136403 (2010).

[30] A. Zazunov, A. L. Yeyati, and R. Egger, Phys. Rev. B 84, 165440 (2011).

[31] C.-H. Lin, J. D. Sau, and S. Das Sarma, Phys. Rev. B 86, 224511 (2012).

[32] E. Prada, P. San-José, and R. Aguado, Phys. Rev. B 86, 180503(R) (2012).
[33] J. Klinovaja, S. Gangadharaiah, and D. Loss, Phys. Rev. Lett. 108, 196804 (2012).

[34] J. Klinovaja and D. Loss, Phys. Rev. B 86, 085408 (2012).

[35] J. S. Lim, L. Serra, R. Lopez, and R. Aguado, Phys. Rev. B 86, 121103 (2012).

[36] J. S. Lim, R. Lopez, and L. Serra, New J. Phys. 14, 083020 (2012).

[37] J. S. Lim, R. Lopez, and L. Serra, Europhys. Lett. 103, 37004 (2013).

[38] L. Serra, Phys. Rev. B 87, 075440 (2013).

[39] J. Osca and L. Serra, Phys. Rev. B 88, 144512 (2013).

[40] X.-J. Liu and A. M. Lobos, Phys. Rev. B 87, 060504 (2013).

[41] M. Lee, H. Khim, and M.-S. Choi, Phys. Rev. B 89, 035309 (2014).

[42] J. Alicea, G. Refael, F. von Oppen, and M. P. A. Fisher, Nat. Phys. 7, 412 (2011).

[43] J. D. Sau, D. J. Clarke, and S. Tewari, Phys. Rev. B 84, 094505 (2011).

[44] B. I. Halperin, Y. Oreg, A. Stern, G. Refael, J. Alicea, and F. von Oppen, Phys. Rev. B 85, 144501 (2012).

[45] J. Klinovaja, P. Stano, and D. Loss, Phys. Rev. Lett. 109, 236801 (2012).

[46] F. Pientka, L. Jiang, D. Pekker, J. Alicea, G. Refael, Y. Oreg, and F. von Oppen, New J. Phys. 15, 115001 (2013).

[47] L. Jiang, D. Pekker, J. Alicea, G. Refael, Y. Oreg, A. Brataas, and F. von Oppen, Phys. Rev. B 87, 075438 (2013).

[48] H. Wu, D. W. L. Sprung, and J. Martorell, Phys. Rev. B 45, 11960 (1992). 